\title{
Initial Validation of Cytokine Measurement by ELISA in Canine Feces
}

\author{
Nathalee Prakash $^{1,2}$, Phil Stumbles $^{2}$, Caroline Mansfield ${ }^{1}$ \\ ${ }^{1}$ School of Veterinary and Biomedical Sciences, Murdoch University, Perth, Australia \\ ${ }^{2}$ Faculty of Veterinary Science, The University of Melbourne, Parkville, Australia \\ Email: cmans@unimelb.edu.au
}

Received July 30, 2013; revised August 30, 2013; accepted September 10, 2013

Copyright (C) 2013 Nathalee Prakash et al. This is an open access article distributed under the Creative Commons Attribution License, which permits unrestricted use, distribution, and reproduction in any medium, provided the original work is properly cited.

\begin{abstract}
Measurement of fecal cytokines has been used as a marker of intestinal inflammation in people and correlates with endoscopic findings. The aim of this study was to evaluate the use of canine-specific enzyme-linked immunosorbant assays (ELISAs) for quantification of cytokines in canine fecal samples as a non-invasive biomarker. Interleukin (IL)-6, $-8,-10,-23 / 12$ p 40 and TNF- $\alpha$ were assessed by using spiked fecal samples from 3 healthy dogs. Standard curve validation was performed, and the impact of time to freeze, duration of storage and number of freeze-thaw cycles on cytokine concentration were also examined. All the cytokines assayed could be detected, with varying accuracy. The mean coefficient of variation (CV) for all standard curves ranged from $2.95 \%-9.8 \%$. The mean intra-assay CV ranged from $3.1 \%-11.14 \%$, and inter-assay CV from $4.36 \%-18.83 \%$. Recovery of IL-23 was poor $(7.23 \%-17.12 \%)$, precluding further interpretation of stability studies. Mean recovery did not appear to be affected by time to freeze and repeat freeze-thaw cycles in all cytokines investigated. Recovery for all cytokines after short-term storage of 30 days at $-80^{\circ} \mathrm{C}$ showed a recovery of $\leq 70 \%$ or $\geq 130 \%$. In conclusion, although fecal IL- $6,-8,-10$, and TNF- $\alpha$ could be used as biomarkers of intestinal inflammation in the dog, the quality of laboratory performance and poor recovery at lower concentrations limit their application. Bench-top and freeze-thaw stability was acceptable, and samples should ideally be analyzed within a week. Investigation involving dogs with acute and chronic inflammatory intestinal disease is required to determine the role of this methodology in a clinical setting.
\end{abstract}

Keywords: Fecal Cytokines; ELISA Validation; Canine; Enteritis

\section{Introduction}

Existing diagnostic procedures to identify intestinal inflammation in dogs are generally expensive or invasive. Histopathology is currently considered the gold standard for diagnosis of active intestinal inflammation, with biopsies obtained endoscopically or surgically. Biopsies, however, are subject to intra- and inter-observer variation and may be of variable sample quality [1-3]. Additionally, in clinical gastroenterology the aim of treatment is to induce remission, which is defined by the American Food and Drug Administration as "an absence of inflammatory symptoms in conjunction with evidence of mucosal healing" [4]. Repeated endoscopy and biopsies are not always permitted in veterinary practice, and current laboratory tests are unable to establish clearly when remission is reached. The uses of disease activity indices aid in monitoring the progress of patients, but themselves have subjectivity in their scoring, or may be influenced by the presence of co-morbidities [5-7].

Fecal biomarkers are a heterogeneous group of substances that leak from, or are generated by, inflamed intestinal mucosa [7]. An ideal fecal biomarker should be non-invasive, reproducible, sensitive, specific, and with clear reference intervals able to distinguish between normal and diseased dogs. A variety of potential markers have been assessed in dogs to date, including calprotectin, Alpha1-proteinase inhibitor and S100A12 [8]. With people, fecal excretion of ${ }^{111}$ Indium-labeled leukocytes currently serves as the gold standard fecal marker of inflammation $[7,8]$. However, its use, along with other techniques such as fecal excretion of ${ }^{51}$ Chromium-labeled red cells or radio-labeled proteins has not been widely adopted in the medical or veterinary field due to issues of radiation exposure and the need for fecal collection over 4 days $[7,8]$. Other fecal markers measure gastrointestinal protein loss, such as alpha1-proteinase inhibitor in dogs, which is protected from intestinal pro- 
teases $[9,10]$. However, these markers are not specific for inflammatory disease $[7,11,12]$. Furthermore, these fecal tests are not widely available [13].

Cytokines are effector proteins that regulate immunity, and are potential biomarkers for diagnostic and therapeutic monitoring being more reflective of general inflamemation. Fecal cytokines such as Tumor Necrosis Factor-Alpha (TNF- $\alpha$ ) have been shown to be a useful marker of disease activity in people with inflammatory bowel disease (IBD) and to correlate well with endoscopic findings $[14,15]$. Fecal measurement of anti-inflammatory cytokines interleukin (IL)- 4 and -10 has been shown to increase with clinical resolution of IBD [16], whilst both IL-2 and interferon (IFN)- $\gamma$ have been shown to significantly increase in people with Norovirus associated diarrhea [17]. As well, IL-8 and IL-1 $\beta$ were increased in some patients with enteroaggregative Escherichia coli infection [18].

It is thought that different factors e.g. bacterial pathogen-associated molecular patterns or activation of tolllike receptors may incite different combinations of cytokines which are predominantly T helper (Th)-1 (e.g. IL-1, -8, TNF- $\alpha$ ) or Th-2 (IL-6, IL-10) mediated [19]. Recent studies in men have shown that a distinct subset of $\mathrm{T}$ helper cells (Th17) drives inflammation and pathology in the human gut. The mechanism by which this occurs is unknown but it is thought to involve a milieu of cytokines including IL-17 and -23 [20,21]. The aim of this study was to investigate the use of enzyme linked immunosorbant assay (ELISA) for detection of IL-6, -8, -10, $-23 / 12 p 40$ and TNF- $\alpha$ in canine fecal samples. As this bioanalytical method has been validated for use with other matrixes in the same species, only a partial validation was performed [22].

\section{Material and Methods}

\subsection{Samples}

Assay validation was performed on fecal samples collected immediately after voiding from three healthy staffowned dogs and consisted of the following breeds: Japanese spitz (female, neutered), Border collie (male, neutered), and a golden retriever (male, neutered). All three dogs were between 2 - 3 years of age and had no history of gastrointestinal signs or weight loss in the 2 months prior to sample collection. All samples were collected in the morning. No medications including NSAIDS, antibiotics or corticosteroids had been administered for at least 3 months prior to sample collection, apart from worming prophylaxis.

\subsection{Collection and Processing of Fecal Samples}

Fecal samples were collected and were kept at $2^{\circ} \mathrm{C}-4^{\circ} \mathrm{C}$ until processed, within an hour of submission. Samples were divided into $1 \mathrm{~g}$ aliquots and placed in polypropylene tubes with $5 \mathrm{~mL}$ of protease inhibitor cocktail P8340 diluted 1:100. The mixture was vortexed for one minute or until the sample was thoroughly homogenized. Samples were then centrifuged at $1200-1500 \mathrm{RCF}$ for 5 minutes at $4^{\circ} \mathrm{C}$, and $0.5 \mathrm{~mL}$ aliquots of the supernatant were separated into polypropylene tubes, stored on dry ice, then at $-80^{\circ} \mathrm{C}$ until assayed. Samples were kept on ice at all other times during processing. Undiluted supernatant samples were spiked with moderately low levels of each cytokine as the validation sample (VS) for validation studies. All three VSs were included with each validation run and were analyzed in duplicate. Assays were performed over several days, with no more than one run per day per ELISA being evaluated. All assays were performed by a single operator (NP).

\subsection{IL-6, -8, -10 and TNF- $\alpha$ Assays}

Canine immunoassays for IL- $6,-8,-10$ and TNF- $\alpha^{2}$ were used according to manufacturer's instruction, with the exception of overnight incubation of fecal samples at $4^{\circ} \mathrm{C}$ to increase sensitivity at the lower limit of detection (LLOD). All samples were run in duplicate.

\subsection{IL-12/23p40 Assay}

An IL-23 immunoassay was developed from a canine IL-12/23p40 assay development kit ${ }^{3}$. The assay employs the quantitative sandwich enzyme immunoassay technique and is performed on 96-well flat-bottom, high-binding plate ${ }^{4}$. The plate was coated with $100 \mu \mathrm{L} /$ well goat anticanine IL-12/23p 40 as the capture anti-canine monoclonal antibody and incubated overnight at $4^{\circ} \mathrm{C}$. Subsequent steps were carried out at room temperature.

The blocking agent and reagent diluent were $1 \% \mathrm{BSA}$ in phosphate buffered saline, wash buffer was $0.05 \%$ Tween 20 in phosphate buffered saline, and the substrate solution a 1:1 mixture of hydrogen peroxide and tetramethylbenzidine. Serial two-fold dilutions were performed on a $4000 \mathrm{pg} / \mathrm{mL}$ recombinant canine IL-12/23p40 standard to generate an eight-point curve. A biotinylated goat anti-canine IL-12/23p40 and streptavidin is used to precipitate a color change that is proportional to the amount of IL-12 and IL-23p40 bound in the initial step. The reaction is stopped by $2 \mathrm{~N}$ sulphuric acid and the optical density of each well is read immediately at $450 \mathrm{~nm}$, and $540 \mathrm{~nm}$.

\subsection{Standard Curve Validation}

All standard curves were prepared using the supplied

${ }^{1}$ Sigma Aldrich, Missouri, USA.

${ }^{2}$ QuantikineTM Canine Immunoassay kits (catalogue numbers CA6000 CA8000, CA1000, CATA00) R\&D Systems, Minneapolis, USA.

${ }^{3}$ Canine IL23/12p40 Duoset (catalogue number DY1969) R\&D Systems, Minneapolis, USA.

${ }^{4}$ Greiner Bio-one, Frickenhausen, Germany. 
cytokine standards that were reconstituted with the reagent diluents to produce a two-fold dilution series on the day of the assay. This produced at least six non-zero standards (excluding blank and anchor points). Back calculations were obtained from six standard curves performed for each cytokine. ELISA validation included description of the standard curves with $\mathrm{CV}$ of the back-calculated values and Square of Pearson's Correlation Coefficient (R2) calculated for each of the cytokines assayed. Acceptance criteria required that the CVs for at least $75 \%$ of the calibration standards should be $<20 \%$ [23].

\subsection{Limits of Detection}

The LLOD was calculated using the mean and standard deviation of the absorbance of the blank samples (assay diluent) to define the lowest concentrations of fecal cytokines that can be reliably distinguished. The LLOD was determined based on manufacturer's guidelines by adding two standard deviations to the mean optical density of the zero standard replicates and calculating the corresponding concentration based on Equation (1) below:

$2 \times$ S.D. absorbance zero standard $(0 \mathrm{pg} / \mathrm{mL}) /$

$\Delta$ absorbance $(0 \mathrm{pg} / \mathrm{mL}$ - lowest standard $\mathrm{pg} / \mathrm{mL})$

$\times$ lowest standard $(\mathrm{pg} / \mathrm{mL})$
The upper limit of detection (ULOD) was measured using the highest standard concentration value measured for that cytokine. Sample analytes of biological systems are predicted not to exceed ULOD based on previous studies on fecal cytokines $[13,17,24]$.

\subsection{Intra-Assay and Inter-Assay Precision}

All three VSs were used to calculate the intra-assay and inter-assay precision. The nominal spiked concentrations can be found in Table 1. Four pairs of each sample were run within the same assay, as well as in duplicates on three separate days. Inter- and intra-assay CV was used as criteria to validate the precision of ligand-binding assays with a CV of $<25 \%$ deemed acceptable [23]. Dilution series on the day of the assay. This produced at least six non-zero standards (excluding blank and anchor points). Back calculations were obtained from six standard curves performed for each cytokine. ELISA validation included description of the standard curves with CV of the back-calculated values and Square of Pearson's Correlation Coefficient $\left(\mathrm{R}^{2}\right)$ calculated for each of the cytokines assayed. Acceptance criteria required that the CVs for at least $75 \%$ of the calibration standards should lie within $20 \%$ [23].

Table 1. Overview of results for initial validation of IL-6, -8, -10, -23 and TNF- $\alpha$ ELISA in 3 dogs.

\begin{tabular}{|c|c|c|c|c|c|}
\hline & IL-6 & IL-8 & IL-10 & IL-23 & TNF- $\alpha$ \\
\hline \multicolumn{6}{|l|}{ Standard Curve Validation } \\
\hline $\begin{array}{l}C V^{*} \text { of standards } \% \\
(\text { mean } \pm \text { std dev })\end{array}$ & $9.80 \pm 4.65$ & $2.95 \pm 3.61$ & $8.08 \pm 3.74$ & $7.84 \pm 5.63$ & $3.25 \pm 2.06$ \\
\hline Mean $\mathbf{R}^{2}$ value & 0.998 & 0.999 & 0.996 & 0.960 & 0.991 \\
\hline $\begin{array}{l}\text { Example of best } \\
\text { fit trendline }\end{array}$ & $y=0.0010 x \pm 0.1102$ & $y=0.0030 x-0.0069$ & $y=0.0028 x \pm 0.0867$ & $y=0.0025 x \pm 0.1564$ & $y=0.0047 x \pm 0.1354$ \\
\hline \multicolumn{6}{|l|}{ Sensitivity } \\
\hline $\mathbf{L L O D}^{\dagger}(\mathbf{p g} / \mathbf{m L})$ & 4.90 & 0.99 & 0.95 & 1.11 & 1.19 \\
\hline $\mathrm{ULOD}^{\ddagger}(\mathrm{pg} / \mathrm{mL})$ & 2000.00 & 1000.00 & 1000.00 & 4000.00 & 500.00 \\
\hline \multicolumn{6}{|l|}{ Validation Samples } \\
\hline $\begin{array}{l}\text { Nominal concentration } \\
\text { of } \mathrm{VS}^{\S} \mathrm{pg} / \mathrm{mL}\end{array}$ & 48.8 & 31.0 & 31.0 & 61.0 & 45.5 \\
\hline $\begin{array}{l}\text { Assayed conc of } \mathrm{VS} \mathrm{pg} / \mathrm{mL} \\
(\text { mean } \pm \text { std dev })\end{array}$ & $81.7 \pm 22.89$ & $24.69 \pm 3.01$ & $11.91 \pm 2.16$ & $10.84 \pm 18.79$ & $23.5 \pm 15.48$ \\
\hline Intra-assay CV (\%) & 3.10 & 4.96 & 3.10 & 11.14 & 4.25 \\
\hline Inter-assay CV (\%) & 11.52 & 18.83 & 4.36 & 6.25 & 4.08 \\
\hline \multicolumn{6}{|c|}{ Accuracy Studies (mean recovery \pm std dev) \% } \\
\hline Low spike (\%) & $109.97 \pm 9,88$ & $66.38 \pm 3.44$ & $83.44 \pm 9.63$ & $17.12 \pm 19.00$ & $65.76 \pm 7.73$ \\
\hline Med spike (\%) & $91.17 \pm 2.3$ & $75.11 \pm 2.92$ & $97.54 \pm 10.33$ & $7.23 \pm 7.42$ & $83.46 \pm 5.18$ \\
\hline High spike (\%) & $100.31 \pm 1.84$ & $86.84 \pm 2.14$ & $104.70 \pm 5.52$ & $14.52 \pm 12.76$ & $90.91 \pm 2.94$ \\
\hline \multicolumn{6}{|c|}{ Stability Studies (mean recovery \pm std dev) \% } \\
\hline 3 hours on bench & $82.03 \pm 3.56$ & $95.95 \pm 1.88$ & $78.92 \pm 7.95$ & NA & $81.22 \pm 10.36$ \\
\hline 3 freeze-thaw cycles & $94.38 \pm 9.06$ & $93.27 \pm 1.36$ & $73.80 \pm 8.07$ & NA & $78.15 \pm 12.71$ \\
\hline 1 month at $-80^{\circ} \mathrm{C}$ & $130.84 \pm 12.79$ & $133.17 \pm 9.84$ & $52.00 \pm 17.81$ & NA & $28.29 \pm 6.40$ \\
\hline
\end{tabular}

${ }^{*}$ Coefficient of variation; ${ }^{\dagger}$ Lower limit of detection; ${ }^{\dagger}$ Upper limit of detection; ${ }^{\S}$ Validation Sample. 


\subsection{Recovery}

Undiluted supernatant samples from all three dogs were used as baselines for the spike and recovery experiments. These samples were spiked with low, moderate and high concentrations of the respective recombinant cytokines. The spiking levels were determined from the assay detection limits, with the low spike being two-fold above the LLOD and the high spike two-fold below the ULOD. Recovery was quantified as a comparison of an observed (assayed) result to its theoretical true value, expressed as a percentage of the nominal (theoretical) concentration (Equation (2)). A recovery of $75 \%-125 \%$ was deemed acceptable [23,25].

$$
\begin{aligned}
& \text { Recovery }(\%) \text { : measured concentration / } \\
& \text { (neat concentration }+ \text { nominal concentration }) \times 100
\end{aligned}
$$

\subsection{Stability Studies}

Short-term storage at room-temperature and $-80^{\circ} \mathrm{C}$, as well as freeze-thaw stability was assessed $[23,25,26]$. Bench-top stability was assessed at room temperature for up to 3 hours, as well as short-term storage stability at $-80^{\circ} \mathrm{C}$ for 1 month. The acceptance criterion was defined as a mean recovery of between $70 \%-130 \%$ [23], compared to their respective reference baseline samples assayed i.e. recovery $=$ (assayed concentration of variable/assayed concentration of baseline sample) $\times 100$. Freeze-thaw stability was assessed for up to three cycles. Freeze/thaw intolerance was defined as a recovery of $<70 \%$ compared to original concentrations.

\section{Results}

An overview of the ELISA validation results for IL-6, -8, $-10,-23$ and TNF- $\alpha$ is shown in Table 1. All cytokines could be detected, with varying accuracy. The mean $\mathrm{CV}$ of the standard curves of IL- $6,-8,-10,-23$, and TNF- $\alpha$ ranged from a minimum of $2.95 \%$ to $9.80 \%$. The $\mathrm{R}^{2}$ obtained for standard curves derived from each assay ranged from 0.960 to 0.999 (mean of 0.988 ). The mean intra-assay CV ranged from $3.10 \%$ to $11.14 \%$, and inter-assay CV from $4.36 \%$ to $18.83 \%$. The recovery of the low spike for IL- 8 and TNF- $\alpha$ were $66.38 \%$ and $65.76 \%$ respectively. There was also poor recovery of IL-23 from fecal samples spiked with IL-23, with a recovery of $7.23 \%-17.12 \%$. This precluded any further interpretation of the test results from the bench-top, storage and stability studies for that particular cytokine. The recovery for all other concentrations for the spike-and-recovery study was more than $75 \%$.

Results for all bench-top and freeze thaw stability studies fell within acceptance criteria of recovery between $70 \%-130 \%$. The mean recovery ranged from
$78.92 \%-95.95 \%$ for samples left for 3 hours on benchtop at room temperature. The mean recovery ranged from $73.80 \%$ - $94.38 \%$ for samples that were subjected to three freeze-thaw cycles. Finally, the samples were found to be intolerant to storage at $-80^{\circ} \mathrm{C}$ for one month, with all cytokines assayed having a mean recovery of $\leq 70 \%$ or $\geq 130 \%$ (range of $28.29-133.17 \%$ ).

\section{Discussion}

There have been several studies using semi-quantitative methods and real-time reverse transcriptase polymerase chain reaction in intestinal biopsy samples to investigate the role of cytokines in mediation of chronic intestinal inflammation in dogs [27-29]. Initial studies showed increased expression of transcripts encoding IL-2, -5, $-12 \mathrm{p} 40$ TNF- $\alpha$ and Transforming Growth Factor- $\beta$ in dogs with inflammatory bowel disease [30,31], but more recent investigations into intestinal cytokine expression have shown no difference between diseased and control samples [27,32]. The choice of cytokines assayed in this study was based on previous fecal cytokine studies in people with acute or chronic enteropathies, assessing both Th1 and Th2 subsets $[14,16,18]$. In addition, IL-23, produced by the distinct subset of T helper cells (Th17), was also included, due to its role in driving inflammation and pathology in the gut [33-35].

Studies have shown undetectable or low concentrations of cytokines in plasma and serum samples from healthy subjects [36,37]. Cytokine IL-2, -4, -5, -10, TNF- $\alpha$, IFN- $\gamma$ and IFN- $\alpha$ concentrations documented in pathogen induced diarrhea ranged from $0.0-51.4 \mathrm{pg} / \mathrm{mL}$ [17]. As preliminary results showed undetectable concentrations in native samples of the cytokines assayed, and fecal cytokine concentrations in diseased dogs have not been documented to date, it was decided that our VSs should be spiked with moderate concentrations of the respective cytokines to mimic concentrations found in human diseased states. The assays were then evaluated based on these VSs for reproducibility and accuracy.

From the results, the reproducibility of the standard curves in the assays was acceptable. An increase in standard deviation and $\mathrm{CV}$ at the lower concentrations was noted. The higher variation in the assay of lower concentrations of cytokine is not unexpected $[23,36]$. However, the clinical implication of this observation is unknown as the presence and degree of fecal cytokine aberrations in dogs with acute and chronic gastrointestinal disease has not yet been documented. Assay accuracy may be affected if concentrations occur at the lower end of the standard curve. Assay precision otherwise appears to be adequate and meets the recommended criteria of $\leq 20 \%$ for intra-assay $\mathrm{CV}$ and $\leq 25 \%$ for inter-assay $\mathrm{CV}$ for validation of ligand-binding assays $[25,38]$. 
In this study, there were significant inconsistencies in cytokine recovery for the low spikes of IL-8 and TNF- $\alpha$. The recovery of IL-23 for all spikes was markedly poor and precluded further interpretation of stability studies. The poor recoveries observed may be due to proteases that were not inactivated by the protease-inhibitor cocktail at initial sample handling, or the presence of nonspecific inhibitors. Both of these effects would be more apparent with lower cytokine concentrations. Unfortunately, effects of other protease inhibitors were not assessed as part of our investigations. Depending on the working concentrations of IL- 8 and TNF- $\alpha$ in a clinical setting, these observations may limit the capacity of these ELISAs to be used as a quantitative test. However, it may still prove useful as a qualitative or semi-quantitative measure of disease activity.

Current recommendations for cytokine measurement are to process and freeze plasma/serum samples within an hour of collection [36]. In this study, recovery was still within acceptable limits of $70 \%-130 \%$ in samples left for three hours at room temperature. Sample stability was also deemed acceptable for up to three freeze-thaw cycles.

Finally, the authors investigated the stability of cytokines over short-term storage. In the clinical and experimental setting, stability over longer term storage is ideal to guarantee confidence in the results obtained. Most cytokines in serum have been shown to be stable for up to 2 years, although IL-6 and IL-10 degraded up to $50 \%$ of baseline values within $2-3$ years at $-80^{\circ} \mathrm{C}$ [36]. Due to the unpredictable effect of proteases in fecal samples, it was decided to re-assay the samples at a 1 month time point, whereby all the cytokines assayed did not fulfill the acceptance criteria of having a recovery between $70 \%-130 \%$. As all other validation assays were performed within a week of collection and processing, and the authors recommend that assays be performed during that time frame. Human studies where TNF- $\alpha$ has been undetected or measured have unfortunately not specified their respective storage times before assay to assess comparison of performance [17,25].

There are multiple limitations to the validation study performed, with the small number of subjects being the major one. Parallelism was also not proven given the negligible cytokine concentrations in the neat samples assayed. However, the authors have decided to proceed with validation as higher endogenous levels of the cytokines are expected in diseased samples. Also, although a spiked sample of the biological matrix was used as a VS to mimic clinical samples; this may differ from an endogenous protein and its behavior with assay performance. Finally, given the unknown endogenous working range, second VS of high concentration should also have been assayed as part of the validation study [23].

\section{Conclusion}

In summary, detection of fecal cytokines (IL-6, -8, -10, and TNF- $\alpha$ ) by ELISA may be of use as non-invasive biomarker of inflammation in the dog, however IL-12/ 23 p40 could not be reliably measured. From the data in this study, the authors propose that clinical fecal samples are processed as soon as possible or within an hour of sample collection and are analyzed within a week. This study provides preliminary information for research in dogs with inflammatory intestinal disease. Further investigation is needed to determine if fecal cytokines can be correlated with clinical signs as a predictor of disease.

\section{REFERENCES}

[1] M. D. Willard, A. E. Jergens, R. B. Duncan, M. S. Leib, M. D McCracken, R. C. DeNovo, et al., "Interobserver Variation among Histopathologic Evaluations of Intestinal Tissues from Dogs and Cats," Journal of the American Veterinary Medical Association, Vol. 220, No. 8, 2002, pp. 1177-1182.

http://dx.doi.org/10.2460/javma.2002.220.1177

[2] M. D. Willard, S. L. Lovering, N. D. Cohen and B. R. Weeks, "Quality of Tissue Specimens Obtained Endoscopically from the Duodenum of Dogs and Cats," Journal of the American Veterinary Medical Association, Vol. 219, No. 4, 2001, pp. 474-479. http://dx.doi.org/10.2460/javma.2001.219.474

[3] M. D. Willard, G. E. Moore, B. D. Denton, M. J. Day, J. Mansell, T. Bilzer, et al., "Effect of Tissue Processing on Assessment of Endoscopic Intestinal Biopsies in Dogs and Cats," Journal of Veterinary Internal Medicine, Vol. 24, No. 1, 2010, pp. 84-89. http://dx.doi.org/10.1111/j.1939-1676.2009.0432.x

[4] S. B. Hanauer, "Inflammatory Bowel Disease," The New England Journal of Medicine, Vol. 334, No. 13, 1996, pp. 841-848.

http://dx.doi.org/10.1056/NEJM199603283341307

[5] K. Allenspach, B. Wieland, A. Grone and F. Gaschen, "Chronic Enteropathies in Dogs: Evaluation of Risk Factors for Negative Outcome," Journal of Veterinary Internal Medicine, Vol. 21, No. 4, 2007, pp. 700-708. http://dx.doi.org/10.1111/j.1939-1676.2007.tb03011.x

[6] A. E. Jergens, C. A. Schreiner, D. E. Frank, Y. Niyo, F. E. Ahrens, P. D. Eckersall, et al., "A Scoring Index for Disease Activity in Canine Inflammatory Bowel Disease," Journal of Veterinary Internal Medicine, Vol. 17, No. 3, 2003, pp. 291-297. http://dx.doi.org/10.1111/j.1939-1676.2003.tb02450.x

[7] A. Poullis, R. Foster, T. C. Northfield and M. A. Mendall, "Review Article: Faecal Markers in the Assessment of Activity in Inflammatory Bowel Disease," Alimentary Pharmacology \& Therapeutics, Vol. 16, No. 4, 2002, pp. 675-681.

http://dx.doi.org/10.1046/j.1365-2036.2002.01196.x

[8] I. Angriman, M. Scarpa, R. D’Incà, D. Basso, C. Ruffolo, L. Polese, et al., "Enzymes in Feces: Useful Markers of 
Chronic Inflammatory Bowel Disease," Clinica Chimica Acta, Vol. 381, No. 1, 2007 pp. 63-68. http://dx.doi.org/10.1016/j.cca.2007.02.025

[9] K. F. Murphy, A. J. German, C. G. Ruaux, J. M. Steiner, D. A. Williams and E. J. Hall, "Fecal Alpha1-Proteinase Inhibitor Concentration in Dogs with Chronic Gastrointestinal Disease," Veterinary Clinical Pathology, Vol. 32, No. 2, 2003, pp. 67-72. http://dx.doi.org/10.1111/j.1939-165X.2003.tb00316.x

[10] C. G. Ruaux, J. M. Steiner and D. A. Williams, "ProteinLosing Enteropathy in Dogs is Associated with Decreased Fecal Proteolytic Activity," Veterinary Clinical Pathology, Vol. 33, No. 1, 2004, pp. 20-22.

http://dx.doi.org/10.1111/j.1939-165X.2004.tb00344.x

[11] N. Berghoff and J. M. Steiner, "Laboratory Tests for the Diagnosis and Management of Chronic Canine and Feline Enteropathies," Veterinary Clinics of North America: Small Animal, Vol. 41, No. 2, 2011, pp. 311-328. http://dx.doi.org/10.1016/j.cvsm.2011.01.001

[12] T. Melgarejo, D. A. Williams and E. K. Asem, "EnzymeLinked Immunosorbent Assay for Canine A1-Protease Inhibitor," American Journal of Veterinary Research, Vol. 59, No. 2, 1998, pp. 127-130.

[13] H. Lettesjö, T. Hansson, C. Peterson, K. A. Ung, G. Ringström, H. Abrahamsson, et al., "Detection of Inflammatory Markers in Stools from Patients with Irritable Bowel Syndrome and Collagenous Colitis," Scandinavian Journal of Gastroenterology, Vol. 41, No. 1, 2006, pp. 54-59. http://dx.doi.org/10.1080/00365520510023909

[14] C. P. Braegger, S. Nicholls, S. H. Murch, S. Stephens and T. T. MacDonald, "Tumour Necrosis Factor Alpha in Stool as a Marker of Intestinal Inflammation," Lancet, Vol. 339, No. 8785, 1992, pp. 89-91. http://dx.doi.org/10.1016/0140-6736(92)90999-J

[15] C. G. Peterson, P. Sangfelt, M. Wagner, T. Hansson, H. Lettesjö and M. Carlson, "Fecal Levels of Leukocyte Markers Reflect Disease Activity in Patients with Ulcerative Colitis," Scandinavian Journal of Clinical \& Laboratory Investigation, Vol. 67, No. 8, 2007, pp. 810-820. http://dx.doi.org/10.1080/00365510701452838

[16] T. Saiki, K. Mitsuyama, A. Toyonaga, H. Ishida and K. Tanikawa, "Detection of Pro- and Anti-Inflammatory Cytokines in Stools of Patients with Inflammatory Bowel Disease," Scandinavian Journal of Gastroenterology, Vol. 33, No, 6, 1998, pp. 616-622. http://dx.doi.org/10.1080/00365529850171891

[17] G. Ko, Z. D. Jiang, P. C. Okhuysen and H. L. DuPont, "Fecal Cytokines and Markers of Intestinal Inflammation in International Travelers with Diarrhea Due to Noroviruses," Journal of Medical Virology, Vol. 78, No. 6, 2006, pp. $825-828$. http://dx.doi.org/10.1002/jmv.20630

[18] D. E. Greenberg, Z. D. Jiang, R. Steffen, M. P. Verenker and H. L. DuPont, "Markers of Inflammation in Bacterial Diarrhea among Travelers with a Focus on Enteroaggregative Escherichia Coli Pathogenicity," The Journal of Infectious Diseases, Vol. 185, No. 7, 2002, pp. 944-949. http://dx.doi.org/10.1086/339617

[19] S. Ardizzone and G. B. Porro, "Inflammatory Bowel Di- sease: New Insights into Pathogenesis and Treatment," Journal of Internal Medicine, Vol. 252, No. 6, 2002, pp. $475-496$ http://dx.doi.org/10.1046/j.1365-2796.2002.01067.x

[20] I. Monteleone, F. Pallone and G. Monteleone, "Interleukin-23 and Th17 Cells in the Control of Gut Inflammation," Mediators of Inflammation, Vol. 2009, 2009, Article ID: 297645. http://www.hindawi.com/journals/mi/2009/297645/

[21] P. J. Morrison, S. J. Ballantyne and M. C. Kullberg, "Interleukin-23 and T Helper 17-Type Responses in Intestinal Inflammation: From Cytokines to T-Cell Plasticity," Immunology, Vol. 133, No. 4, 2011, pp. 397-408. http://dx.doi.org/10.1111/j.1365-2567.2011.03454.x

[22] US Department of Health and Human Services Food and Drug Administration, "Guidance for Industry: Bioanalytical Method Validation,” Rockville, 2001.

[23] M. A. Valentin, S. Ma, A. Zhao A, F. Legay and A. Avrameas, "Validation of Immunoassay for Protein Biomarkers: Bioanalytical Study Plan Implementation to Support Preclinical and Clinical Studies," Journal of Pharmaceutical and Biomedical Analysis, Vol. 55, No. 5, 2011, pp. 869-877. http://dx.doi.org/10.1016/j.jpba.2011.03.033

[24] M. A. Sidler, S. T. Leach and A. S. Day, "Fecal S100a12 and Fecal Calprotectin as Non-invasive Markers for Inflammatory Bowel Disease in Children," Inflammatory Bowel Diseases, Vol. 14, No. 3, 2008, pp. 359-366. http://dx.doi.org/10.1002/ibd.20336

[25] J. W. Lee, V. Devaranarayan, Y. C. Barrett, R. Weiner, J. Allinson, S. Fountain, et al., "Fit-for-Purpose Method Development and Validation for Successful Biomarker Measurement," Pharmaceutical Research, Vol. 23, No. 2, 2006, pp. 312-328. http://dx.doi.org/10.1007/s11095-005-9045-3

[26] K. Brookes, J. Cummings, A. Backen, A. Greystoke, T. Ward, G. C. Jayson, et al., "Issues on Fit-For-Purpose Validation of a Panel of ELISAs for Application as Biomarkers in Clinical Trials of Anti-Angiogenic Drugs," British Journal of Cancer, Vol. 102, No. 10, 2010, pp. 1524-1532.

[27] A. E. Jergens, I. M. Sonea, A. M. O'Connor, L. K. Kauffman, S. D. Grozdanic, M. R. Ackermann, et al., "Intestinal Cytokine mRNA Expression in Canine Inflammatory Bowel Disease: A Meta-Analysis with Critical Appraisal," Comp Med, Vol. 59, No. 2, 2009, pp. 153-162.

[28] N. Nguyen Van, K. Taglinger, C. R. Helps, S. Tasker, T. J. Gruffydd-Jones and M. J. Day, "Measurement of Cytokine mRNA Expression in Intestinal Biopsies of Cats with Inflammatory Enteropathy Using Quantitative RealTime RT-PCR," Veterinary Immunology and Immunopathology, Vol. 113, No. 3-4, 2006, pp. 404-414. http://dx.doi.org/10.1016/j.vetimm.2006.06.010

[29] I. R. Peters, C. R. Helps, E. L. Calvert, E. J. Hall and M. J. Day, "Cytokine mRNA Quantification in Histologically Normal Canine Duodenal Mucosa by Real-Time RTPCR," Veterinary Immunology and Immunopathology, Vol. 103, No. 1-2, 2005, pp. 101-111. http://dx.doi.org/10.1016/j.vetimm.2004.08.020

[30] A. J. German, C. R. Helps, E. J. Hall and M. J. Day, 
"Cytokine mRNA Expression in Mucosal Biopsies from German Shepherd Dogs with Small Intestinal Enteropathies," Digestive Diseases and Sciences, Vol. 45, No. 1, 2000, pp. 7-17.

http://dx.doi.org/10.1023/A:1005436721798

[31] A. E. Ridyard, T. J. Nuttall, R. W. Else, J. W. Simpson and H. R. Miller, "Evaluation of Th1, Th2 and Immunosuppressive Cytokine mRNA Expression within the Colonic Mucosa of Dogs with Idiopathic Lymphocytic-Plasmacytic Colitis," Veterinary Immunology and Immunopathology, Vol. 86, No. 3-4, 2002, pp. 205-214. http://dx.doi.org/10.1016/S0165-2427(02)00039-9

[32] I. R. Peters, C. R. Helps, E. L. Calvert, E. J. Hall and M. J. Day, "Cytokine mRNA Quantification in Duodenal Mucosa from Dogs with Chronic Enteropathies by RealTime RT-PCR," Journal of Veterinary Internal Medicine, Vol. 19, No. 5, 2005, pp. 644-653. http://dx.doi.org/10.1111/j.1939-1676.2005.tb02742.x

[33] M. Sarra, F. Pallone, T. T. MacDonald and G. Monteleone, "Il-23/Il-17 Axis in IBD," Inflammatory Bowel Diseases, Vol. 16, No. 10, 2010, pp. 1808-1813. http://dx.doi.org/10.1002/ibd.21248

[34] W. Shen and S. K. Durum, "Synergy of Il-23 and Th17 Cytokines: New Light on Inflammatory Bowel Disease,"
Neurochemical Research, Vol. 35, No. 6, 2010, pp. 940946. http://dx.doi.org/10.1007/s11064-009-0091-9

[35] H. H. Uhlig, B. S. McKenzie, S. Hue, C. Thompson, B. Joyce-Shaikh, R. Stepankova, et al., "Differential Activity of Il-12 and Il-23 in Mucosal and Systemic Innate Immune Pathology," Immunity, Vol. 25, No. 2, 2006, pp. 309-318. http://dx.doi.org/10.1016/j.immuni.2006.05.017

[36] W. De Jager, K. Bourcier, G. T. Rijkers, B. J. Prakken and V. Seyfert-Margolis, "Prerequisites for Cytokine Measurements in Clinical Trials with Multiplex Immunoassays" BMC Immunology, Vol. 10, 2009, pp. 52-63.

[37] N. Aziz, P. Nishanian, R. Mitsuyasu, R. Detels and J. L. Fahey, "Variables That Affect Assays for Plasma Cytokines and Soluble Activation Markers" Clinical and Diagnostic Laboratory Immunology, Vol. 6, No. 1, 1999, pp. 89-95.

[38] B. DeSilva, W. Smith, R. Weiner, M. Kelley, J. Smolec, B. Lee, et al., "Recommendations for the Bioanylytical Method Validation of Ligand Binding Assays to Support Pharmacokinetic Assessments of Macromolecules," Pharmaceutical Research, Vol. 20, No. 11, 2003, pp. 18851900.

http://dx.doi.org/10.1023/B:PHAM.0000003390.51761.3 $\underline{\mathrm{d}}$ 